\title{
$\$$ Research Square

\section{Genetic Algorithm Based Oversampling Approach to Prune the Class Imbalance issue in Software Defect Prediction}

Arun Chinnathambi ( $\square$ arunchinnathambi@gmail.com )

SRM Institute of Science and Technology

Lakshmi C

SRM Institute of Science and Technology

\section{Research Article}

Keywords: Class Imbalance, software fault prediction, synthetic samples, generating samples of minority class, over sampling techniques, Genetic algorithm, false alarm rate, evolutionary algorithm

Posted Date: April 26th, 2021

DOl: https://doi.org/10.21203/rs.3.rs-434360/v1

License: (c) (i) This work is licensed under a Creative Commons Attribution 4.0 International License.

Read Full License 


\section{Abstract}

Class Imbalance is the potential problem that has been existent in machine learning, which hinders the performance of the classification algorithm when applied in real world applications such as electricity pilferage, fraudulent transactions, anomaly detection, prediction of rare diseases, etc. Class Imbalance refers to the problem where the distribution of the sample is skewed or biased towards one particular class. Due to its intrinsic nature the software fault prediction dataset falls into the same category where the software modules contain fewer defective modules compared to the non-defective modules. Majority of the over sampling techniques that has been proposed is to address the issue by generating synthetic samples of minority class to balance the dataset. But the synthetic samples generated are near duplicates that also results in over-generalization issue. We thus propose a novel oversampling approach to introduce synthetic samples using Genetic algorithm (GA). GA is a form of evolutionary algorithm that employs biologically inspired techniques such as inheritance, mutation, selection, and crossover. The proposed algorithm generates synthetic sample of minority class based on the distribution measure and ensures that the samples are diverse within the class and are efficient. The proposed over sampling algorithm has been compared with SMOTE,B-SMOTE, ADASYN, Random Oversampling, MAHAKIL and no sampling approach with a 20 defect prediction dataset from the Promise repository and five prediction models. The results indicate that the Genetic algorithm over sampling approach improves the fault prediction performance and reduced false alarm rate.

\section{Full Text}

This preprint is available for download as a PDF.

\section{Figures}

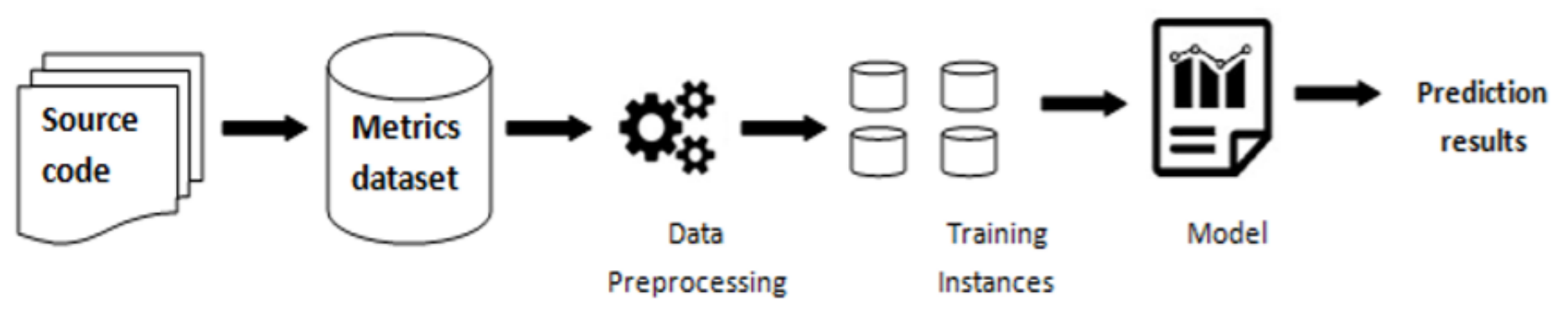

Figure 1

Software Defect Prediction using Machine learning Model 

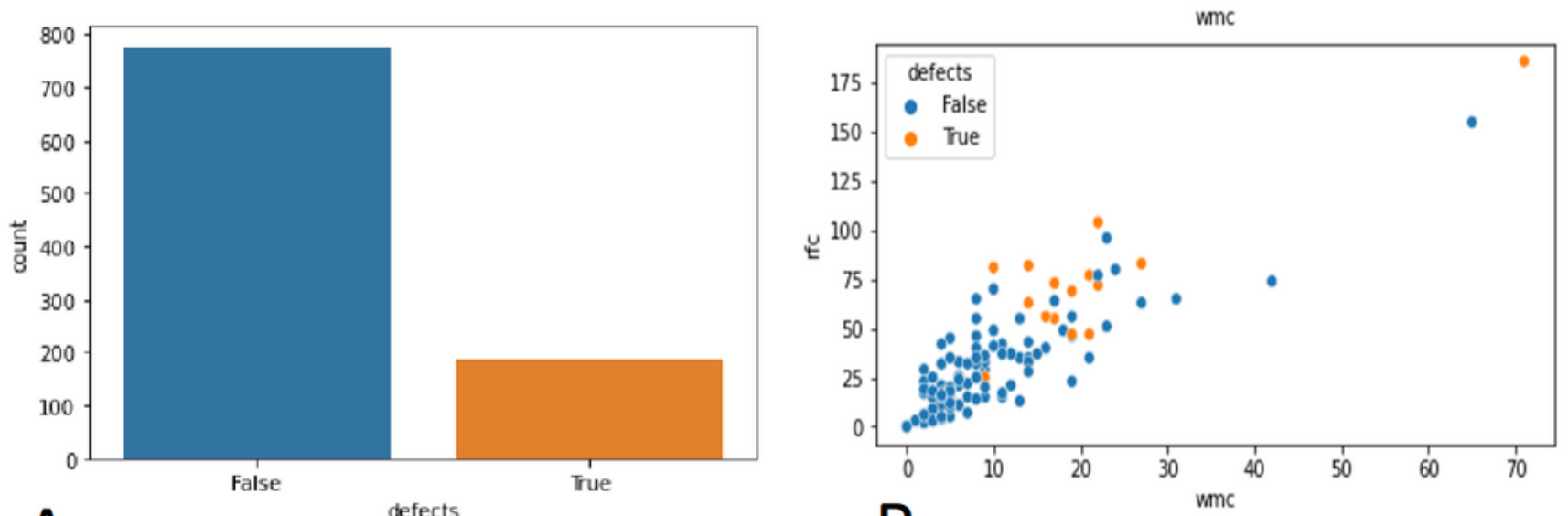
A
defects
B
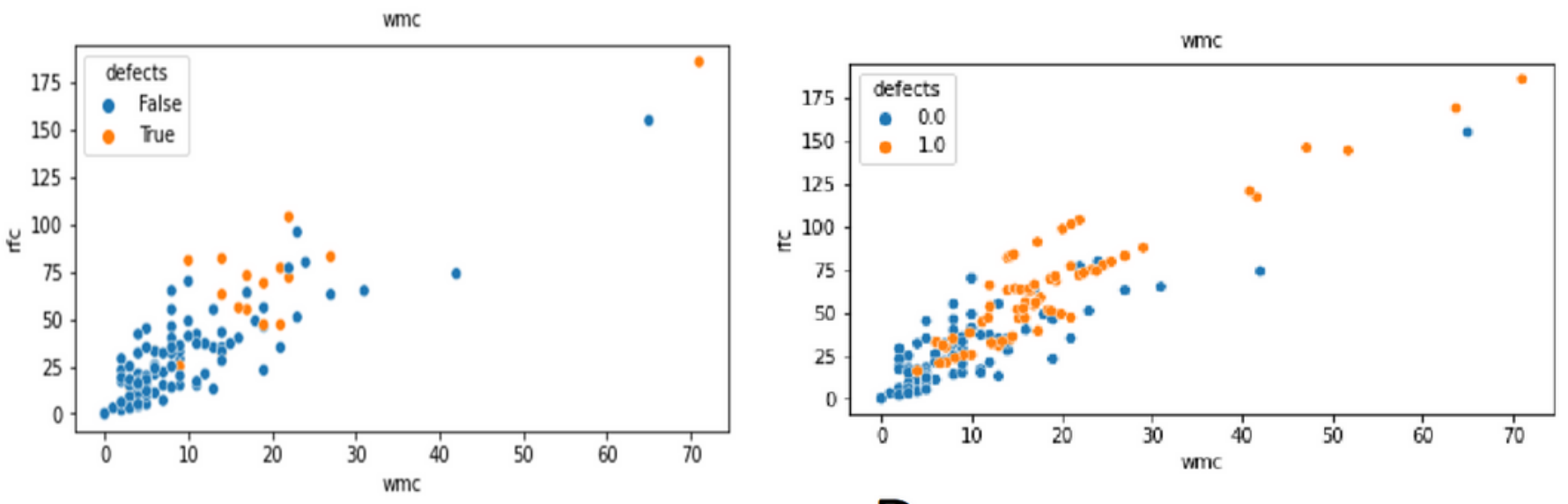

C

D


$\mathrm{E}$

\section{Figure 2}

a Distribution of the class Imbalance Data b Distribution of data after sampling by ROS c Distribution of data after Sampling using ROS d Distribution of data after sampling using SMOTE e Distribution of data after sampling using BSMOTE $f$ Distribution of data after sampling using ADASYN 

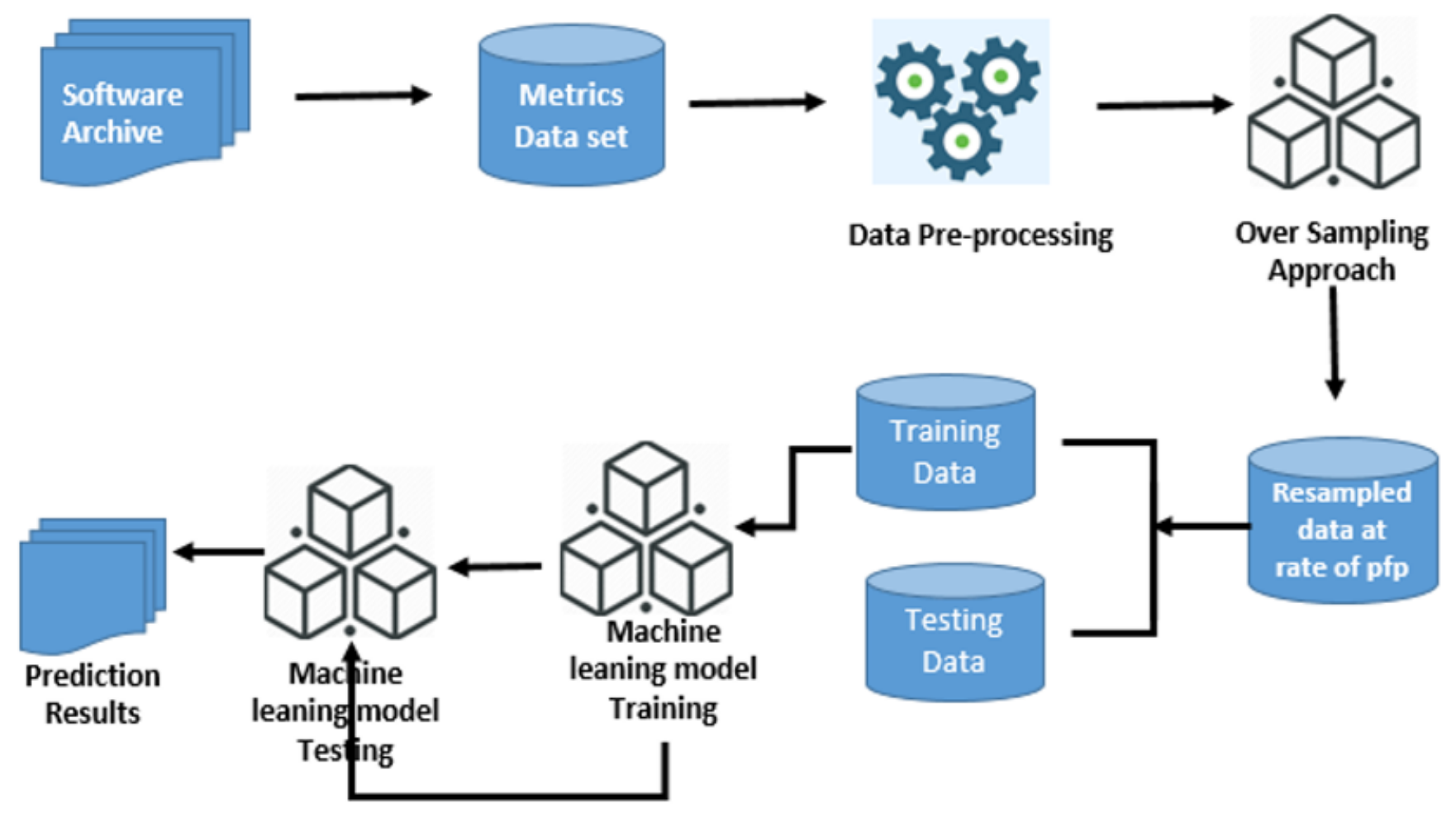

\section{Figure 3}

\section{Experimental Framework}

Performance of Oversampling method using different machine learning model

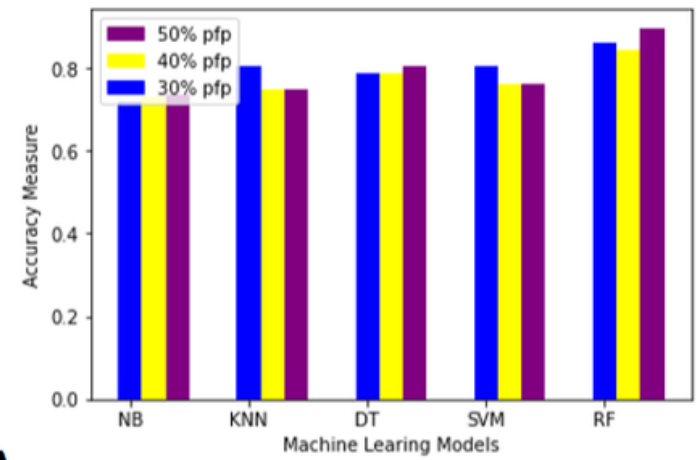

A

Performance of Oversampling method using different machine learning model

Performance of Oversampling method using different machine learning model
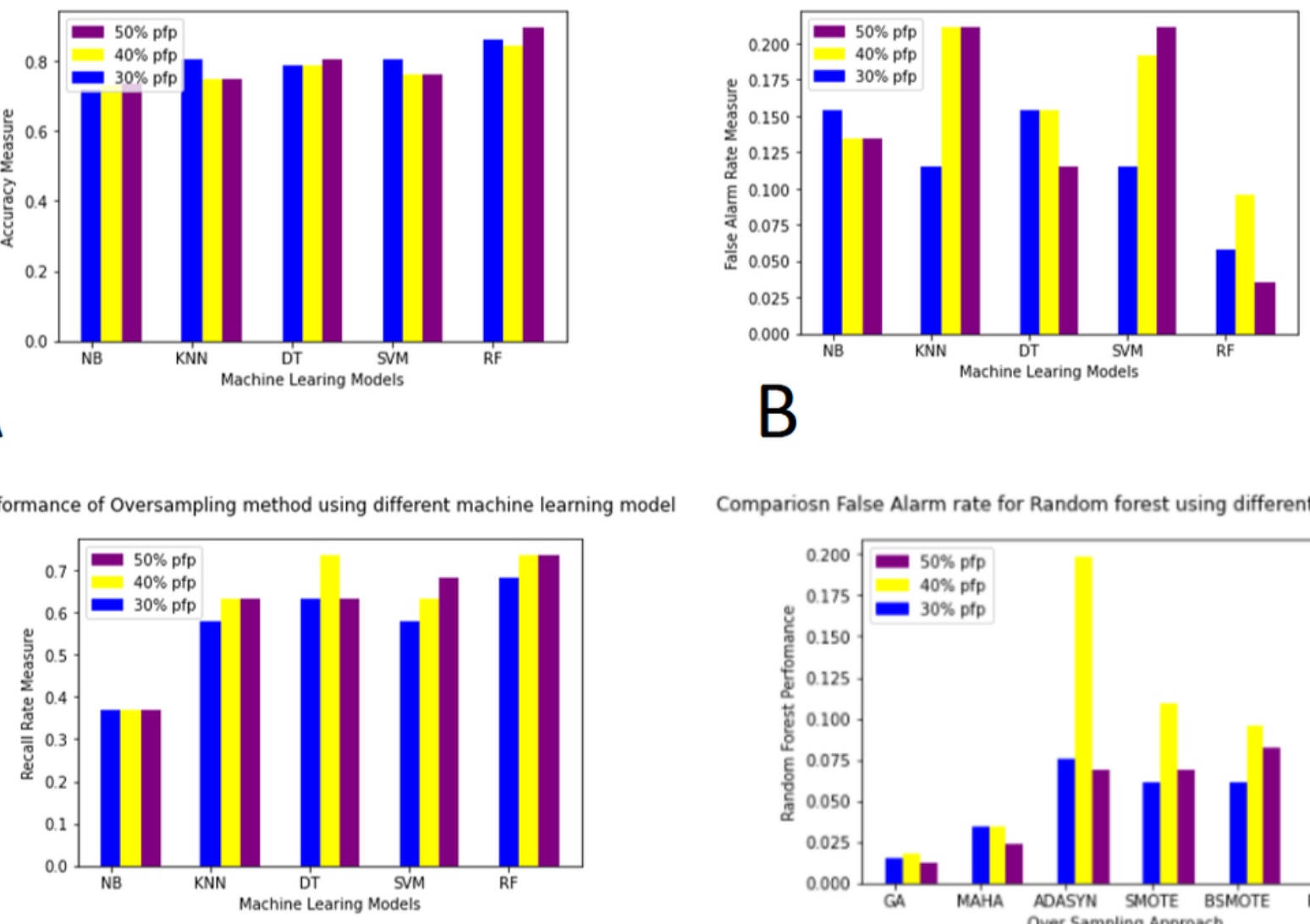

C

Compariosn False Alarm rate for Random forest using different sampling techniques

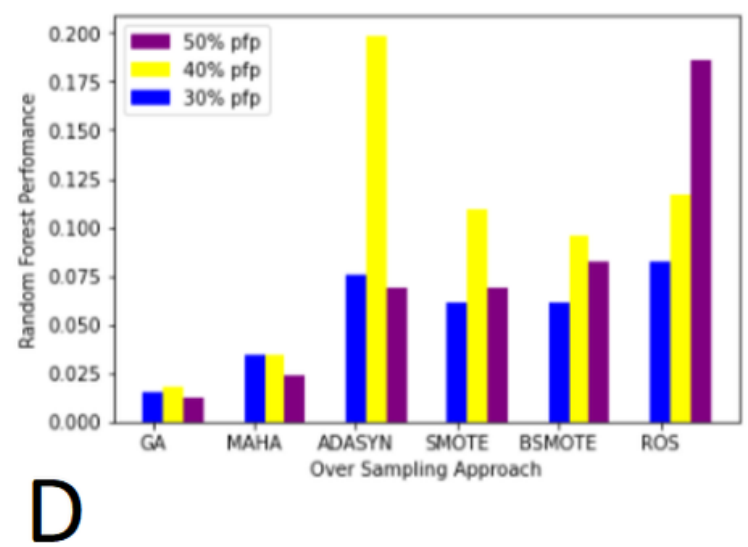


Figure 4

a Performance comparison of over sampling approaches using different prediction models based on accuracy b Performance comparison of over sampling approaches using different prediction models based on false alarm rate $c$ Performance comparison of over sampling approaches using different prediction models based on recall $d$ Comparison of false alarm rate of proposed Oversampling technique with others at each pfp levels

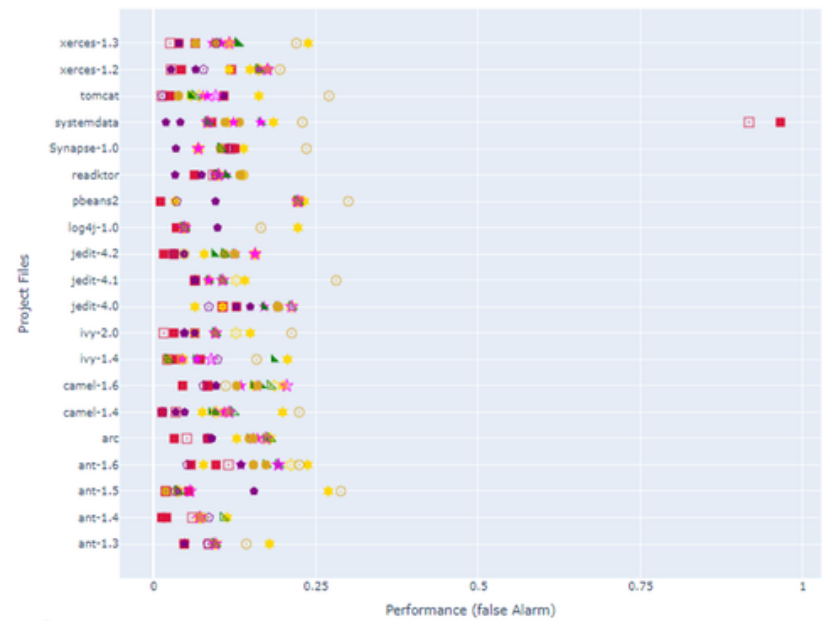

A
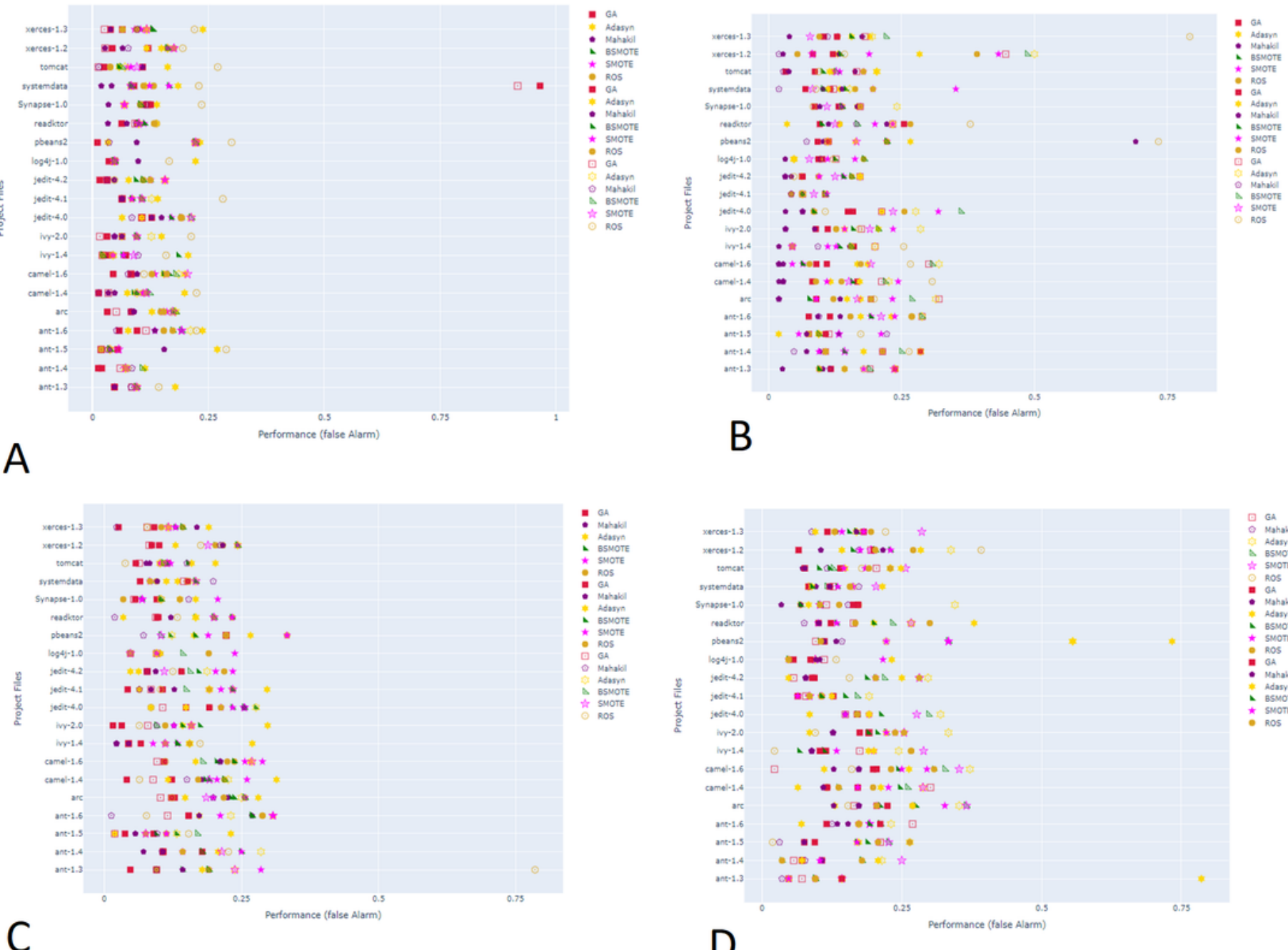

C

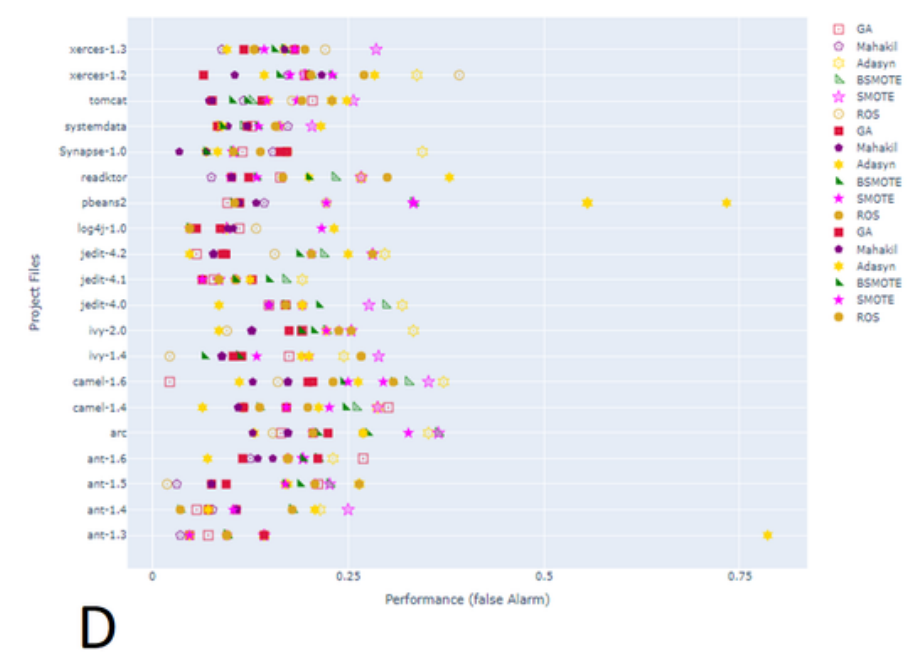

$E$

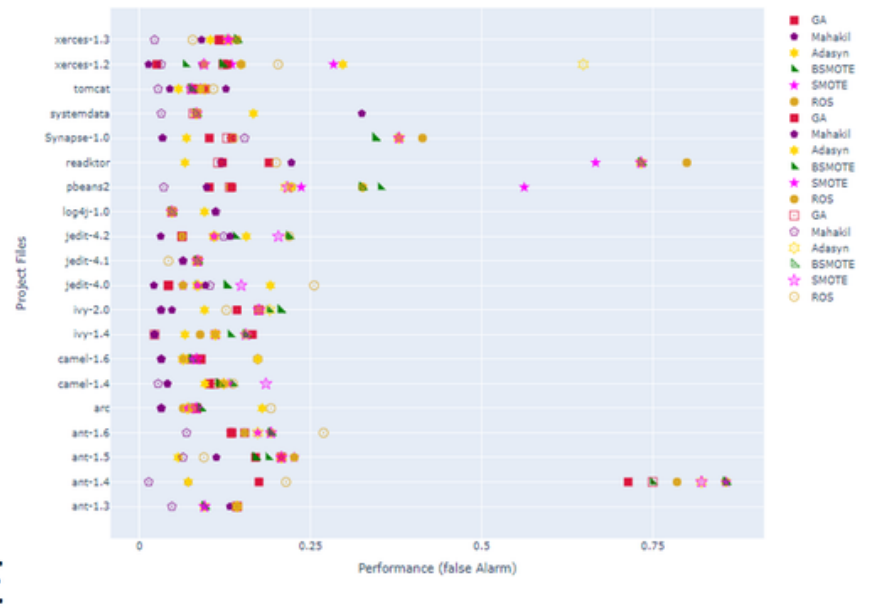




\section{Figure 5}

a Comparison on the performance Random Forest model on different over sampling techniques Comparison on the performance Support Vector Machine model on different over sampling techniques Comparison on the performance C4.5 model on different over sampling techniques Comparison on the performance KNN model on different over sampling techniques Comparison on the performance NB model on different over sampling techniques 